\title{
Updated BTS Adult Bronchiectasis Guideline 2018: a multidisciplinary approach to comprehensive care
}

\author{
Adam T Hill, ${ }^{1}$ Sally A Welham, ${ }^{2}$ Anita L Sullivan, ${ }^{3}$ Michael R Loebinger ${ }^{4}$
}

\author{
ABSTRACT \\ The new British Thoracic Society (BTS) \\ Guideline for Bronchiectasis in Adults has been \\ published. This article provides a summary of \\ key highlights of the new BTS Guideline and \\ covers who to investigate, what investigations \\ should be carried out and a management \\ strategy.
}

\section{INTRODUCTION}

Internationally, bronchiectasis is increasingly common with prevalence rates currently around 5 per 1000 in the UK population. ${ }^{1}$ Data from primary care have confirmed comorbid disease in two-thirds of patients, with asthma and chronic obstructive pulmonary disease (COPD) being the most common comorbidities in $42 \%$ and $36 \%$, respectively. ${ }^{1}$ The Bronchiectasis Severity Index international scoring system has been developed to assess prognosis, with increased mortality in patients with severe bronchiectasis. ${ }^{2}$

The new British Thoracic Society (BTS) Guideline for Bronchiectasis in Adults covers adult patients with clinically significant (non-cystic fibrosis) bronchiectasis, that is, patients with the clinical syndrome of bronchiectasis with radiological confirmation. ${ }^{3}$ A full summary of all recommendations and good practice points has been published separately. ${ }^{4}$

It is based on the best available evidence and the methodology used adheres strictly to the criteria as set by the AGREE II (Appraisal of Guidelines for Research and Evaluation) collaboration. ${ }^{5}$ It provides a timely update to the first BTS Bronchiectasis guideline published in $2010 .^{6}$ The guideline has new sections including a quick guide, a stepwise management plan, a stepwise plan for physiotherapy management, how to manage the deteriorating

\footnotetext{
${ }^{1}$ Department of Respiratory Medicine, Royal Infirmary of Edinburgh, Edinburgh, UK

${ }^{2}$ British Thoracic Society, London, UK

${ }^{3}$ Department of Respiratory Medicine, Queen Elizabeth Hospital, Birmingham, UK

${ }^{4}$ Host Defence Unit, Division of Respiratory Medicine, Royal Brompton Hospital, London, UK
}

Correspondence to Professor Adam T Hill, Department of Respiratory Medicine, Royal Infirmary of Edinburgh, Edinburgh, Scotland EH16 4SA, UK; adam.hill318@nhs.net patient and research recommendations provided in a PICO format.

\section{DIAGNOSIS AND INVESTIGATIONS}

Investigation for bronchiectasis is recommended in patients with persistent production of mucopurulent or purulent sputum, particularly with relevant associated risk factors. COPD is a common comorbidity in bronchiectasis and in a recent BTS National Bronchiectasis Audit, COPD was found to be present in around 24\%. ${ }^{7}$ The guidelines recommend considering bronchiectasis in patients with COPD with frequent exacerbations (two or more annually) and/or positive sputum cultures for potentially pathogenic micro-organism (including Pseudomonas aeruginosa) while stable.

Investigation for bronchiectasis should include a baseline chest X-ray, which is a useful first-line test to exclude other pathologies, and a thin section CT of the chest, as the chest X-ray lacks both sensitivity and specificity in the diagnosis of bronchiectasis.

As comorbidities and associated conditions are frequently present, comorbidities and medical history should be recorded. Tests recommended for all patients have focused on aetiologies that have specific therapies that would alter bronchiectasis management and include allergic bronchopulmonary aspergillosis, immunodeficiency and ciliary defects. Full blood count, serum total immunoglobulin (Ig)E and specific IgE or skin prick test to Aspergillus should be measured in all patients to investigate for allergic bronchopulmonary aspergillosis. Serum $\operatorname{IgG}, \operatorname{IgA}$ and $\operatorname{IgM}$ should be measured to exclude immunodeficiency and baseline specific antibody levels against capsular polysaccharides of Streptococcus pneumoniae to investigate for specific antibody deficiency. Patients with low pneumococcal antibodies should be immunised with the 23 valent polysaccharide pneumococcal vaccine, followed by repeat measurement of specific antibody levels 4-8 weeks later. Discussion with an immunologist is recommended, if levels remain suboptimal, in case regular replacement subcutaneous or intravenous $\operatorname{IgG}$ therapy is needed.
Cystic fibrosis (CF) testing should be performed according to National Institute for Health and Care Excellence Guidelines for CF in patients with supporting clinical features ${ }^{8}$ and primary ciliary dyskinesia (PCD) should be investigated in patients with supporting clinical features. ${ }^{9}$ Measurement of nasal nitric oxide is the recommended first-line investigation for PCD.

\section{MONITORING}

The guideline group has provided updated guidance on recommended monitoring of patients with mild and more severe bronchiectasis. For mild bronchiectasis all should have:

1. Baseline: severity score, such as the Bronchiectasis Severity Index; thin-section chest CT scan; sputum for bacterial and mycobacterial culture; assessment for aetiology; assessment of comorbidities, baseline spirometry and oxygen saturation.

2. Annually: body mass index; assessment of exacerbations; sputum for bacterial culture; Medical Research Council (MRC) dyspnoea score; spirometry; oxygen saturation.

3. The deteriorating patient: repeat thin-section chest CT scan; sputum for bacterial and mycobacterial culture; assessment for aetiology; assessment of comorbidities.

For more severe bronchiectasis, the same applies except there should be 6 monthly assessment of exacerbation history, MRC dyspnoea score, oxygen saturation on room air and sputum for bacterial culture.

There is a lack of randomised controlled trials proving the efficacy of regular chest clearance, but the guideline group felt this should be first-line management of all patients. All patients should see a respiratory physiotherapist to be taught the active cycle breathing technique and consider gravity-assisted positioning. The frequency of chest clearance will be advised by the physiotherapist, but in more advanced disease, at least two times per day will be recommended, with sessions lasting from 10 to a maximum of $30 \mathrm{~min}$. There should be a review within 3 months. If the above is not effective or there is poor adherence, oscillation positive expiratory pressure devices and forced expiratory technique should be considered. Nebulised isotonic $(0.9 \%$ saline $)$ or hypertonic $(3 \%$ and above) saline should also be considered. BTS and European Respiratory Society (ERS) guidelines recommend not to use nebulised DNAse in view of strong 


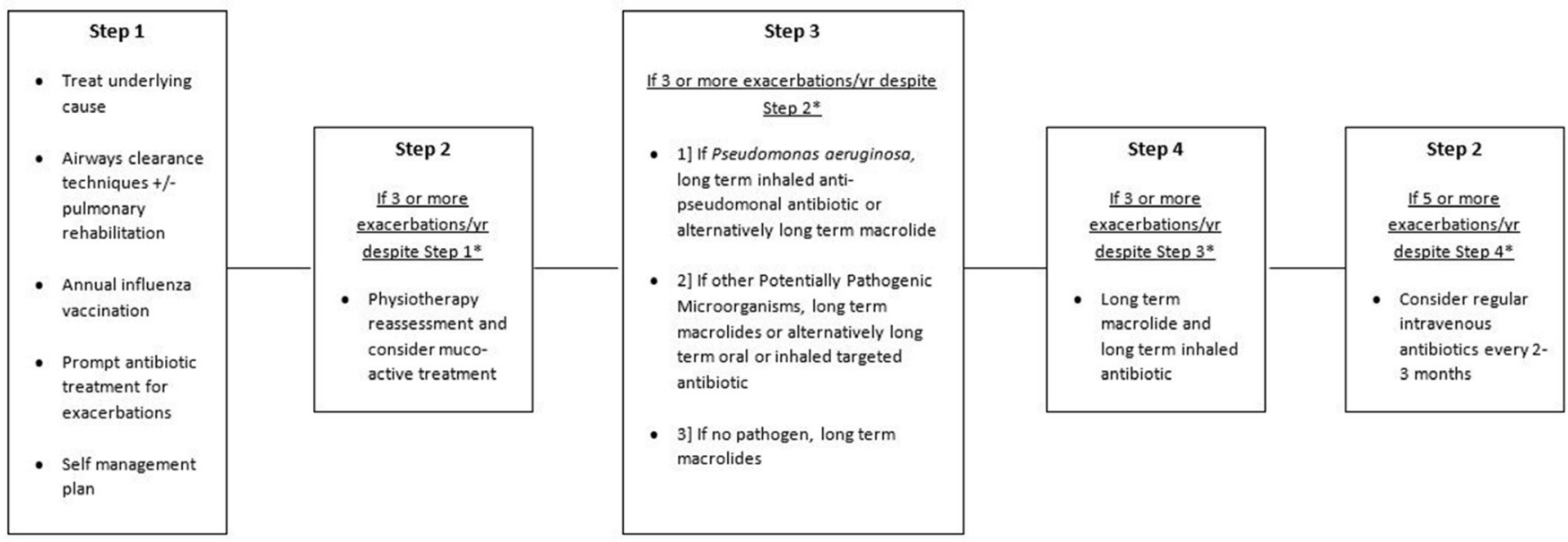

*Consider this step if significant symptoms persist despite previous step, even if not meeting exacerbation criteria

\begin{abstract}
Figure 1 Stepwise management
Antibiotics are used to treat exacerbations that present with an acute deterioration (usually over several days) with worsening local symptoms (cough, increased sputum volume or change of viscosity, increased sputum purulence with or without increasing wheeze, breathlessness, haemoptysis) and/or systemic upset. The flow diagram refers to three or more annual exacerbations.
\end{abstract}

Figure 1 Stepwise management.

evidence that this treatment leads to a poor outcome. ${ }^{310}$

\section{STEPWISE MANAGEMENT}

A key improvement of this guideline is the development of a stepwise management plan (see figure 1). Step 1 considers five key elements for all patients: treating an underlying cause if identified; performing airways clearance techniques; annual influenza vaccination; a self-management plan (eg, on the BTS website ${ }^{11}$ ); and the prompt use of appropriate antibiotics for exacerbations when required. Antibiotics are used to treat exacerbations that present with an acute deterioration (usually over several days) with worsening local symptoms (cough, increased sputum volume or change of viscosity, increased sputum purulence with or without increasing wheeze, breathlessness, haemoptysis) and/or systemic upset and should be given without waiting for sputum culture results, as this can lead to a delay in the management of the exacerbation.

If patients are suffering three or more exacerbations per year despite this, they should be reviewed again by the respiratory physiotherapist, as in practice, patients often are poorly compliant with regular chest clearance. Mucoactive treatments can be considered at this stage. Steps 3 and 4 consider the addition of single or combined long-term antibiotic treatment (figure 1) in patients with three or more exacerbations per year despite optimisation and gives guidance based on their known sputum bacteriology status. Due to the evidence available, for inhaled antibiotic therapies, the first-line inhaled antipseudomonal antibiotic recommended is colistin ${ }^{12}$ and second line is inhaled gentamicin, ${ }^{13}$ pending new phase III trials. Patients who remain very symptomatic with high exacerbation burdens $(\geq 5$ per year) can be considered for regular intravenous antibiotic therapy every $2-3$ months.

Another new section in the guideline refers to the management of the deteriorating patient, defined as one with significant and prolonged deterioration of symptoms, unexpected increased frequency or severity of exacerbations, frequent hospital admissions, early relapse after treatment of an exacerbation or rapid decline in lung function. A broad approach is suggested and includes

1. Assessment: assessment of patient understanding; exclusion of specific aetiologies (in particular CF, allergic bronchopulmonary aspergillosis, gastro-oesophageal reflux disease, common variable immunodeficiency and inflammatory bowel disease and repeat testing should be considered in the deteriorating patient); assessment of disease progression with CT chest, lung function tests and oxygen saturation; reassessment of sputum pathogens (bacteria, fungal and mycobacteria) and consideration of induced sputum or bronchoalveolar lavage; assessment of comorbidities (eg, pulmonary hypertension, sinus disease, pulmonary embolism).

2. Optimisation: airway clearance reviewed by the respiratory physiotherapist to assess compliance, regimen, need for pulmonary rehabilitation and mucoactive treatment; exacerbations treatment reviewed.

3. Management: consideration of intravenous antibiotic therapy or a long-term antibiotic regimen and assessment for long-term oxygen therapy, non-invasive ventilation, surgery, transplantation or end of life care, as appropriate.

Secondary care follow-up is recommended in patients with: chronic P. aeruginosa, methicillin-resistant Staphylococcus aureus colonisation or non-tuberculous mycobacteria; deteriorating bronchiectasis with declining lung function; recurrent exacerbations ( $\geq 3$ per year); long-term antibiotic therapy (oral, inhaled or nebulised); associated rheumatoid arthritis, immune deficiency, inflammatory bowel disease, PCD and allergic bronchopulmonary aspergillosis; advanced disease and those considering transplantation.

The guidelines are a timely addition to the literature and also highlight areas for audit and for future research.

Contributors ATH led the development of the article. All authors were involved in the final editing and gave 
approval for the final document.

Funding The authors have not declared a specific grant for this research from any funding agency in the public, commercial or not-for-profit sectors.

Competing interests All authors completed the annual Declaration of Interest form in line with BTS policy. ATH has declared funding from Bayer Healthcare, Boehringer Ingelheim. MRL has declared funding from Bayer Healthcare, Insmed, Pulmocide, Griffols, Polyphor, Savara.

Provenance and peer review Not commissioned; internally peer reviewed.

(c) Author(s) (or their employer(s)) 2019. No commercial re-use. See rights and permissions. Published by BMJ.

\section{D) Check for updates}

To cite Hill AT, Welham SA, Sullivan AL, et al. Thorax 2019;74:1-3.

Received 9 August 2018

Revised 1 November 2018

Accepted 5 November 2018

\section{(S) Linked}

- http://dx.doi.org/10.1136/thoraxjnl-2018-212463

Thorax 2019;74:1-3

doi:10.1136/thoraxjnl-2018-212468

\section{REFERENCES}

1 Quint JK, Millett ER, Joshi M, et al. Changes in the incidence, prevalence and mortality of bronchiectasis in the UK from 2004 to 2013: a population-based cohort study. Eur Respir J 2016:47:186-93.

2 Chalmers JD, Goeminne P, Aliberti S, et al. The bronchiectasis severity index. An international derivation and validation study. Am J Respir Crit Care Med 2014;189:576-85.

3 Hill AT, et al. BTS Guideline for bronchiectasis in adults. Thorax 2019:74:1-3.

4 Hill AT, et al. BTS Guideline for bronchiectasis in adults summary of recommendations, BMJ Open Respiratory Research. 2018

5 AGREE II. https://www.agreetrust.org/agree-ii/

6 Pasteur MC, Bilton D, Hill AT. British Thoracic Society guideline for non-CF bronchiectasis. Thorax 2010:65(Suppl 1):i1-58.
7 Hill AT. British thoracic society national audit report: Adult and paediatric bronchiectasis audits. 2017. https://www.brit-thoracic.org.uk/document-library/ audit-and-quality-improvement/audit-reports/btsbronchiectasis-audits-combined-report-2017/

8 NICE Guideline Cystic Fibrosis: diagnosis and management. 2017. NG78.

9 Lucas JS, Barbato A, Samuel A, et al. European respiratory society guidelines for the diagnosis of primary ciliary dyskinesia. European Respiratory Journal 2016;42(Issue 2).

10 Polverino E, Goeminne PC, McDonnell MJ, et al. European Respiratory Society guidelines for the management of adult bronchiectasis. Eur Respir $\rfloor$ 2017:50:1700629.

11 BTS Quality Standards for clinical significant bronchiectasis in adultsAppendix 1, Self Management Plan. 2012. https://www.brit-thoracic.org.uk/ standards-of-care/quality-standards/bts-bronchiectasisquality-standards/

12 Haworth CS, Foweraker JE, Wilkinson P, et al. Inhaled colistin in patients with bronchiectasis and chronic Pseudomonas aeruginosa infection. Am I Respir Crit Care Med 2014:189:975-82.

13 Murray MP, Govan JR, Doherty CJ, et al. A randomized controlled trial of nebulized gentamicin in non-cystic fibrosis bronchiectasis. Am J Respir Crit Care Med 2011;183:491-9. 